Mots. Les langages du politique

\title{
Peuples autochtones. La fabrique onusienne d'une identité symbolique
}

Indigenous peoples. The construction of a symbolic identity at the United Nations

Pueblos indígenas. La fábrica de una identidad simbólica en las Naciones Unidas Irène Bellier et Veronica González-González

\section{(2) OpenEdition}

Édition électronique

URL : https://journals.openedition.org/mots/22050

DOI : $10.4000 /$ mots. 22050

ISSN : 1960-6001

Éditeur

ENS Éditions

\section{Édition imprimée}

Date de publication : 6 octobre 2015

Pagination : $131-150$

ISBN : $978-2-84788-727-3$

ISSN : 0243-6450

\section{Référence électronique}

Irène Bellier et Veronica González-González, «Peuples autochtones. La fabrique onusienne d'une identité symbolique », Mots. Les langages du politique [En ligne], 108| 2015, mis en ligne le 06 octobre 2017, consulté le 22 avril 2022. URL : http://journals.openedition.org/mots/22050 ; DOI : https:// doi.org/10.4000/mots.22050 


\title{
Peuples autochtones. La fabrique onusienne d'une identité symbolique
}

\author{
You have a home at the United Nations... \\ We begin a new chapter in the history of indigenous \\ peoples at the United Nations. \\ Kofi Annan, Secrétaire général de l’ONU, 2002
}

Comme en témoigne l'adresse de l'ancien Secrétaire général des Nations unies, Kofi Annan, prononcée à l'Instance permanente sur les questions autochtones en mai $2006^{1}$, l'institution du monde dont la charte commence par ces mots : «Nous, peuples... », a accueilli les représentants des dits peuples autochtones pour entendre leurs revendications et y porter réponse (Daes, 2008; Morin, 2006 ; Muehlebach, 2001; Niezen, 2003). Dans le cadre de cet article, nous souhaitons montrer la puissance symbolique des éléments qui ont contribué à créer un espace propice à la négociation d'une identité globale, à travers et pardelà la production d'un instrument de grande valeur morale, politique et symbolique : la Déclaration des Nations unies sur les droits des peuples autochtones (ci-après : «la Déclaration»).

Kofi Annan verbalise la nature du lien symbolique qui rattache les peuples autochtones au système international : la notion de «maison » au sens d'un foyer accueillant (a home) en étant la première marque. Il évoque l'ouverture d'un «nouveau chapitre de l'histoire», lequel inclut dorénavant des peuples longtemps considérés comme «sans histoire »² et il nomme le sujet de cette histoire

1. Cette étude s'appuie sur une recherche financée par le Conseil européen de la recherche dans le cadre du $7^{\mathrm{e}}$ programme-cadre (FP7/2007-2013 Grant Agreement ERC $n^{\circ}$ 249236). Le programme de recherche SOGIP (Échelles de gouvernance - les Nations unies, les États et les Peuples autochtones; l'autodétermination au temps de la globalisation), dirigé depuis 2010 par Irène Bellier (Principal Investigator ERC-SOGIP), est réalisé aux Nations unies et dans une dizaine de pays avec une équipe de jeunes chercheurs dont Veronica González-González (www.sogip.ehess.fr).

2. Ont été considérés comme "sans histoire» les peuples à tradition orale, pourvus ou non de restes archéologiques, sans archives ni élites reconnues. L'ethnohistoire permet de mieux connaître le passé de ces sociétés, mais leur inscription dans les récits nationaux, lorsqu'elle existe, est toujours problématique.

CNRS, EHESS, Institut interdisciplinaire d'anthropologie du contemporain (IIAC), Laboratoire d'anthropologie des institutions et des organisations sociales (LAIOS), ERC-SOGIP

ibellier@ehess.fr

EHESS, Scales of Governance - The UN and Indigenous Peoples (SOGIP)

veronica.gonzalez@ehess.fr 
- indigenous peoples, ou peuples autochtones dans la traduction française agréée, c'est-à-dire un sujet collectif. La puissance symbolique de ce collectif (Bellier, 2012; Dahl, 2009) se niche dans le $s$ de peoples qui établit, en anglais, une différence entre «population » (people) et «peuple» (peoples) : seul le peuple est susceptible de recevoir la personnalité juridique, en droit international.

Le processus politique, ancré dans des luttes juridiques, s'est accompagné de la production de symboles s'appuyant sur une narration historique, l'usage de nouveauxvocables, une rhétorique propre au langage international, un univers de signes (images et logos) qui établissent un pont entre les mondes autochtones et le monde onusien. Cette symbolique converge vers deux phénomènes. Le premier correspond à la mise en visibilité d'une catégorie générique, politique et relationnelle, celle de «peuples autochtones» (Bellier, 2012, 2013a). Le second éclaire le rôle des représentants autochtones dans le scénario international, leur objectif étant de redéfinir la place des entités qu'ils représentent dans les systèmes juridiques et constitutionnels nationaux.

Cet article vise à décrire le processus de production de symboles que l'Onu a avalisé dans le domaine des peuples autochtones, ainsi que les termes de leur reconnaissance et de leur circulation externe. La première partie s'appuie sur nos recherches en anthropologie politique, conduites aux Nations unies depuis 2001, pour démontrer que la construction d'un espace pour les peuples autochtones dans le système international passe par des pratiques symboliques et langagières qui visent à poser les termes de nouveaux paradigmes politiques3. Les représentants autochtones parviennent à influencer un milieu ordonné par les protocoles des États et des instances onusiennes, en s'appuyant sur des références à la coutume, des formes cérémonielles, des logiques d'assemblée uniques et le contrôle d'un vocabulaire qui devient un enjeu politique. On observe une sorte de connivence entre deux formes d'universalisme : l'universalisme des droits humains (inaliénables, interdé-

3. Irène Bellier suit au plan scientifique les rendez-vous annuels de quatre organes des Nations unies spécialisés, pour des sessions de travail d'une à deux semaines : à Genève, de 2002 à 2006, le Groupe de travail sur les populations autochtones, créé en 1982, et le Groupe de travail sur le projet de Déclaration, créé en 1995, dissous après l'adoption de la Déclaration par le Conseil des droits de l'homme en 2006; à New York, depuis 2002, l'Instance permanente sur les questions autochtones; à Genève, depuis 2008, le Mécanisme d'experts sur les droits des peuples autochtones. Ce suivi implique de participer aux sessions plénières, aux «événements parallèles » et aux assemblées autochtones (caucus) qui sont informelles pour les Nations unies mais essentielles à la construction du mouvement autochtone international et de sa base de savoirs partagés. Les caucus préparent les nouveaux venus à la connaissance de l'ONU et aux techniques d'intervention en séance. S'y rendent les autochtones et les ONG, mais pas les fonctionnaires des secrétariats onusiens, ni les représentants des États, sauf invitation expresse (Bellier, 2007). Veronica González-González a mené durant plusieurs années une recherche au sein de plusieurs agences spécialisées et organes de l'ONU (l'Unesco, la FAO, le Haut-Commissariat aux droits de l'homme, l'Instance permanente sur les questions autochtones) pour analyser les manières dont ils traitent les questions liées aux peuples autochtones depuis leur fondation. L'observation participante des terrains onusiens implique l'analyse des documents qui circulent à chacune des réunions et entre celles-ci, comme celle des textes institutionnels ou informels archivés. 
pendants, indivisibles et imprescriptibles) et l'universalisation d'une catégorie «peuples autochtones» qui transcende les spécificités locales.

Dans la seconde partie, qui s'appuie sur des recherches que nous menons sur la circulation des normes relatives aux peuples autochtones, nous identifions certaines tensions impliquées par l'usage de ces symboles. Inspirées du courant théorique du constructivisme social, ces recherches montrent que les symboles ont forgé les fondements d'une nouvelle relation entre les peuples autochtones, les Nations unies et les États. Sur la base de cette rencontre, qui se produit au siège des Nations unies et dans différentes agences spécialisées, se construit un rapport d'interdépendance par lequel le mouvement autochtone international et l'Onu se légitiment mutuellement. Mais le sens que les symboles que nous présenterons ci-après veulent faire circuler, franchit difficilement les frontières des instances dédiées aux questions autochtones, ce qui témoigne des limites de l'alliance entre l'ONU et les peuples autochtones que nous évoquerons en conclusion.

\section{La production de symboles avalisés par les Nations unies}

La production de symboles relatifs aux peuples autochtones s'appuie sur une histoire, sur la mobilisation et la participation des acteurs autochtones et sur une dynamique institutionnelle qui scelle l'alliance établie avec l'Onu (Bellier, 2006a, 2012). Deux symboles puissants sont la production d'une identité commune et l'inscription de celle-ci dans un document de portée universelle. Cela nous conduit à problématiser le phénomène d'institutionnalisation des questions autochtones dans le système international.

\section{L'accord sur l'identité «peuples autochtones»}

La dynamique autochtone initiée à la fin des années soixante-dix vise la reconnaissance de droits pour des populations anciennement ou toujours dominées par l'État colonial. Elle suppose l'établissement d'une base d'intelligibilité de ce que l'expression "peuples autochtones» recouvre et l'identification de la nature des problèmes auxquels se confrontent lesdites populations. Cet effort s'est appuyé sur une étude pionnière menée par le professeur de sociologie équatorien José Martinez Cobo, nommé en 1971 rapporteur spécial des Nations unies sur la discrimination à l'encontre des populations autochtones par l'ancienne Commission aux droits de l'homme. Il s'est poursuivi avec la réalisation d'études réalisées par les membres de la Sous-Commission de la lutte contre les mesures discriminatoires et de la protection des minorités 4 , siégeant au Groupe

4. Cette instance a changé son nom en 1999 pour celui de Sous-Commission de la promotion et la protection des droits de l'homme. Elle a disparu, en 2006, dans le cadre de la réforme qui s'est traduite par la mise en place du Conseil des droits de l'homme. 
de travail mis en place en 1982, sur des thématiques exemplaires telles que «La souveraineté permanente des populations autochtones sur la terre, le territoire et les ressources naturelles» (Daes, 2004) ou la question des «Traités, accords et autres arrangements constructifs » (Martínez, 1999). L'étude de la discrimination témoigne de deux caractéristiques principales. D’une part, elle traite de populations considérées comme «distinctes et spécifiques», par leur mode de vie, leur langue, leur organisation sociale et leur exclusion. Les cinq volumes, produits entre 1973 et 1986, donnèrent lieu à une définition de travail qui pose les bases d'une approche multicritère, laquelle se révèle propice à remettre en question le paradigme essentialiste qui entoure l'identification de «l'indigène ». D'autre part, le partage des informations à l'échelle du monde mit en évidence la nature commune des processus de discrimination auxquelles ces populations étaient (et sont) confrontées, ce qui conduisit à ancrer le traitement de leurs problèmes dans le paradigme des droits fondamentaux. Alors que la notion de « différence » est venue se combiner à l'étude des moyens de remédier à l'exclusion sociopolitique, la scène de l'égalité de droit - entre peuples nationaux et peuples autochtones - a mis face à de nouvelles demandes les États et les agences internationales qui, durant les trente dernières années, se sont profilées dans une gouvernance du monde (Unesco, Pnud, FAO, Banques de développement, etc.).

Le processus relie les scènes nationales à la scène des Nations unies sur laquelle les représentants des peuples autochtones ont fait irruption pour témoigner de leur situation en déployant, face à la politique des États, «la politique du cœur» (Brysk, 2002). Cela a contribué à améliorer la connaissance des mécanismes de marginalisation socio-économique, légale et politique, et à valider une construction identitaire capable de se substituer à la pluralité des désignations locales, tombées en désuétude dans le répertoire linguistique international. L'observation des travaux onusiens montre comment s'effacent, dans les discours comme dans les rapports, les références aux identités singulières, et nos analyses permettent de fournir une explication pratique de ce processus : 1 ) les modes de nomination usités par les États sont contestés localement et globalement en tant que symboles de discrimination. Depuis plusieurs années, les peuples se réapproprient leurs noms en langue vernaculaire, comme en témoignent les Maihuna (Êtres humains-collectif) avec qui l. Bellier a travaillé au Pérou, précédemment connus sous les noms péjoratifs de Orejones (Grandes oreilles) et Koto (du nom du singe hurleur en Amazonie), mais aussi les San, anciennement Bushmen, les Wixarika, anciens Huichol, les Samis, anciens Lapons, etc.; 2) les noms des différents peuples représentés aux Nations unies (soit 4500 des 6000 langues reconnues dans le monde, d'après l'Unesco, 2003) sont intraduisibles dans les langues de travail de l'Onu (anglais, arabe, chinois, espagnol, français et russe) et ils sont difficilement prononcés par les autres, autochtones ou non, que l'on entend bafouiller. Cela conforte l'usage de l'expression «peuples autochtones / indi- 
genous peoples / pueblos indígenas» dans le registre référentiel et indexical; 3) l'expression permet de rendre compte des engagements collectifs d'individus qui ne portent pas la voix de leur communauté d'origine mais celle de tous.

Cet effacement relève aussi d'une stratégie qui prit corps dans les instances représentatives des peuples autochtones, nommément les caucus/cónclaves ou assemblées générales (Bellier, 2007), au sein desquels s'est produit depuis le début des années quatre-vingt un immense travail de médiation des sens et de mise à niveau des participants. Au sein de ces instances circule sans réserve l'expression indigenous peoples / pueblos indigenas / peuples autochtones. Celle-ci a permis aux organisations autochtones d'affirmer une nature non subordonnée au niveau international, en revendiquant des droits qui étaient réservés aux États, tels celui de participer aux négociations de haut niveau et le droit des peuples à disposer d'eux-mêmes. Au niveau local, cela les conduit à contester la légitimité de l'État à prendre des décisions concernant les territoires autochtones, en présence notamment de mégaprojets d'infrastructure routière, hydro-électriques ou miniers : c'est l'un des motifs pour lesquels les organisations autochtones représentatives recourent à la Commission puis à la Cour interaméricaine des droits de l'homme.

Le droit à l'autodétermination, reconnu en droit international, est, à propos des populations autochtones, l'objet d'interprétations continues engageant la question de la souveraineté, laquelle n'est attribuée qu'aux États dans le système onusien. La reconnaissance de l'expression «peuples autochtones» engage ainsi une question essentielle, à deux volets :1) quelle est la nature des peuples qui n'ont pas cédé leur souveraineté volontairement, certains d'entre eux ayant signé des traités attestant de relations de nation à nation ?;2) quelle place occupent-ils dans le modèle de l'État-nation qui se trouve au défi d'intégrer des composantes multiculturelles? Une tierce question hantait les esprits au moment de rédiger la Déclaration : quel motif autre que la discrimination et le racisme permet de tenir ces populations à l'écart de la citoyenneté?

Nous ne répondrons pas à ces questions dans cet article pour nous focaliser sur un point crucial qu'est la formation d'une voix collective autochtone, hautement improbable si l'on songe à la dispersion des sujets, à leurs différences linguistiques et à leur pauvreté relative. Cette voix s'est forgée dans la dynamique de participation, les représentants autochtones (qui ne maîtrisaient rien des protocoles onusiens) important à l'Onu les outils d'une solidarité pratique qui transforma en force collective leurs faiblesses relatives par rapport aux appareils d'État. En contrepartie, l'extrême diversité des peuples autochtones est devenue gérable à partir des formes auto-organisées par les délégués autochtones : assemblées générales, déclarations communes, sélection de représentants régionaux (Bellier, travaux cités).

Les manières d'être, de faire et de dire autochtones surprirent les fonctionnaires onusiens et les délégués des États. Nous ne détaillerons pas ici le choc 
des regards et des pratiques que l'introduction à l'ONU d'une coiffe à plume, de pratiques de fumigations, de tambours, de chants, de vêtements très colorés représenta dans les années quatre-vingt. Au-delà des marques d'une différence visible d'avec le monde des États, l'ensemble autochtone démontre par ces pratiques deux faits essentiels : ses composantes expriment la diversité du monde; sa résistance s'ancre dans ce qu’il dénomme «spiritualité», que l'on comprendra ici comme l'ontologie qui éclaire des cosmovisions distinctes de celles du monde occidental, ainsi que les fondements de systèmes politiques et juridiques qu'il entend faire reconnaître. L'expression de cette diversité s'est combinée à l'apprentissage des manières de faire de la maison-hôte. La maîtrise du protocole et l'acquisition du vocabulaire international sont la face visible d'un processus de façonnage, les autochtones étant mis en situation de s'approprier des outils mis à leur disposition5. À partir de ces différents éléments, la diplomatie des délégations autochtones permit que leurs délégués soient reconnus par les États comme des interlocuteurs admissibles.

L'expression «peuples autochtones » s'est alors imposée, comme une identité porteuse d'une telle puissance symbolique qu'une autre bataille, livrée par les autochtones, s'est engagée au quotidien pour éviter la référence à d'autres termes ou expressions tels que population, communauté, minorité ethnique, race nationale ou tribu. En témoigne la déclaration de Winona LaDuke lors de la Conférence des Nations unies sur les femmes, à Pékin :

Tout comme je suis membre d'une nation de peuples autochtones, il existe des millions d'autochtones dans le monde. Environ 500 millions aujourd'hui. Nous sommes les peuples de la Cordillère, les Maori de Nouvelle-Zélande, nous sommes à Timor Leste, nous sommes les Wara wara d'Australie, les Lakota, les Tibétains, les peuples d'Hawai'i, de Nouvelle-Calédonie, des nations de peuples autochtones. Peuples autochtones. Nous ne sommes pas des populations, pas des minorités, nous sommes des peuples. Nous sommes des nations de peuples. Dans le droit international, nous répondons aux critères de l'État-nation, en possédant un système économique commun, une langue, un territoire, une histoire, une culture et des institutions de gouvernement. Malgré cela les nations autochtones ne sont pas autorisées à participer aux Nations unies. ${ }^{6}$

La catégorie est devenue un outil qui n'est pas simplement rhétorique puisque le terme peuple engage un principe du droit international7. Combi-

5. Par exemple : le Haut-Commissariat aux droits de l'homme assure des programmes de formation des leaders autochtones; le Centre de Documentation pour les peuples autochtones (DoCip) fournit un Secrétariat technique, des ressources documentaires et des interprètes pour faciliter les réunions entre autochtones depuis 1978 ; les agences des Nations unies ne font plus aucune réunion relevant de ce champ de problématiques sans inviter des experts autochtones.

6. Winona LaDuke était à l'époque présidente du Réseau des femmes autochtones, directrice des programmes pour l'environnement du Seventh Generation Fund. Voir http://www.ratical.org/ co-globalize/WinonaLaDuke/Beijing95.html, consulté le 27 janvier 2015.

7. Ce principe est exprimé à l'article 1 commun aux deux Pactes internationaux sur les droits civils et politiques et sur les droits économiques et sociaux (1966) : «Tous les peuples ont le droit de 
née à des formes propres aux discours militants et réinvestie dans les déclarations des autochtones qui mobilisent les vocables frère et sœur (et peu celui de camarade), l'expression établit les bases d'une «communauté horizontale» (Bellier, 2012, p. 74). À partir de la scène internationale, s’identifie une sorte de «communauté globale autochtone» dont l'horizon est plus proche de la «communauté imaginée» par Benedict Anderson (1991) que de la communauté de sang ou d'origine qui renvoie à des processus classificatoires étatiques.

On assiste à l'émergence d'une communauté d'acteurs non étatiques qui s'exprime sur la scène du monde et se dote d'images attestant de cette qualité. En témoigne un logo qui inscrit dans l'emblème des Nations unies (la couronne de laurier), un visuel figurant la réconciliation, soit deux mains de couleurs distinctes sur un fond de références aux entités de la cosmovision autochtone, «classiques » car considérées comme d'importance vitale. Ces entités - l'eau, la terre, l'air et le feu - évoquées par les couleurs et le graphisme, sont invoquées nominalement dans les discours d'ouverture et de clôture des travaux.

\section{Logo de l'Instance permanente des Nations unies sur les questions autochtones}

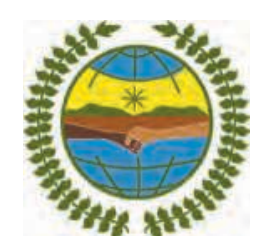

Le récit d'Augusto Willemsen Díaz, le fonctionnaire international qui réalisa avec Martinez-Cobo l'étude précitée sur la discrimination à l'encontre des populations autochtones, illustre cette alliance :

Dans mon pays, le Guatemala, la présence des peuples autochtones est millénaire. Ils sont déterminés à maintenir et à protéger leur identité différente, leurs cultures, coutumes, traditions et institutions propres, ainsi que leurs terres et territoires, qui furent objets de tentatives d'usurpation et de dépossession. Ils en appellent, en outre, au respect. Alors que je travaillais dans le domaine des droits de l'homme, je pensai qu'il me fallait peut-être travailler à la reconnaissance et au respect des droits des peuples autochtones, d'un point de vue plus proche de leurs désirs et de leurs aspirations. Il me parut particulièrement important que soit respectée leur vision collective particulière du cosmos et d'eux-mêmes, leur vision du monde différente, notamment le respect de la Terre-Mère et de la nature, d'importance vitale pour eux. Confiant que le succès était possible, je me dévouai à cette cause, tout en remplissant mes obligations de fonctionnaire du Secrétariat général des Nations unies. (Willemsen Díaz, 2013, p. 22)

disposer d'eux-mêmes. En vertu de ce droit, ils déterminent librement leur statut politique et assurent librement leur développement économique, social et culturel.» 
Le logo figure sur le site de l'Instance permanente. Mais les personnes autochtones se font, elles, photographier à proximité du logo original des Nations unies (la couronne de laurier entourant un planisphère) qui domine la tribune de l'Assemblée générale. Comme s’il fallait par la photographie signaler la présence du sujet dans l'univers onusien, tandis que le dessin a pour fonction de figurer la «terre-mère» (et ses composantes) envers qui les autochtones construisent une filiation. Mais sur le plan juridique, l'Onu est la matrice (la maison) des droits autochtones, ce que l'usage du symbole de la couronne de laurier rappelle.

\section{Une Charte des droits des peuples autochtones}

Prendre place dans un système défini par les États membres de l'Onu représenta un défi pour les délégations autochtones qui, au départ de leurs mobilisations, se présentaient comme des «victimes de l'histoire», en rappelant comment l'histoire de la colonisation avait entravé leur destin. À travers la saisine des secteurs des droits de l'homme, la mobilisation des «organisations de soutien ${ }^{8}$ et l'implication d'experts engagés dans la défense d'une «cause " 9 , s'est engagé un processus de production de normes de droit qui eut pour particularité d'impliquer les sujets qu'il concernait dans la définition de l'instrument négocié (Crawhall, 2011). Nous renvoyons le lecteur vers les travaux consacrés à l'élaboration, à la négociation et à l'adoption de la Déclaration des droits des peuples autochtones (Daes, 2008 ; Charters, Stavenhagen, 2009; Bellier, 2005; Hartley, Joffe, Preston, 2010 ; Allen, Xanthaki, 2011). Mais nous soulignons deux enjeux de cette Déclaration, au-delà de l'accolade du terme "peuples» à celui d' "autochtones». Le premier est le fait de poser des droits collectifs comme moyens de protection. Cela met en relief l'importance de reconnaître les dispositifs sociaux nécessaires à la reproduction d'identités, de langues et de modes de vie, autrement que par le jeu «naturel » des politiques, assimilationnistes, multiculturelles ou néolibérales. Le second enjeu est de ne pas produire des droits nouveaux et spécifiques à une catégorie sociale, ce qui viendrait atténuer la portée universelle des droits de l'homme auxquels se rattachent les droits des peuples autochtones. Ceux-ci complètent le dispositif des droits individuels de l'homme et ne s'y substituent pas.

La Déclaration rassemble les droits existant dans une série de traités, textes, conventions, pactes et déclarations relatifs à la souveraineté, aux droits de la personne, aux droits civils, politiques, économiques, sociaux et culturels, aux droits de la femme, de l'enfant et à la lutte contre la discrimination et

8. L'expression désigne les ONG qui apportent un soutien logistique, financier (et intellectuel) aux délégations et individus autochtones.

9. Qu'il s'agisse de violation de droits humains, de philanthropie ou de cause environnementale, la notion de cause soutient la notion de plaidoyer (advocacy) qui légitime l'action des porteurs de voix sur la scène internationale. 
le racisme. Ses 46 articles s'organisent autour de problématiques nodales en vue de remédier à la discrimination, de réparer les torts de la colonisation, de respecter les entités dont la souveraineté est affirmée dans l'article 3: «Les peuples autochtones ont le droit à l'autodétermination. En vertu de ce droit, ils déterminent librement leur statut politique et assurent librement leur développement économique, social et culturel.»

Les dispositions s'organisent autour : d'un bloc de droits relatifs à la terre, au territoire et aux ressources naturelles; des principes de reconnaissance opératoires dans les domaines de la justice, de la santé, de l'éducation, de la communication; de l'idée de la réparation, de la remise à niveau ou de la compensation pour les dommages passés (et ceux qui pourraient advenir). Soulignons ici un élément très symbolique de la nature de l'engagement qui s'est noué lors de l'adoption de la Déclaration. Les articles sont pour la plupart rédigés sous la forme d'un premier alinéa, «Les peuples autochtones ont le droit de... », suivi d'un second alinéa, «les États doivent... / mettent en place... / veillent... ». Cette écriture distribue un système de reconnaissance et de responsabilité que les Cours des droits de l'homme peuvent interpréter pour résoudre les contentieux dont elles sont saisies (Price, 2012).

La consubstantialité de la relation entre certaines populations, des modes de vie ou de subsistance économique et des cultures s'est axée sur la relation à la Terre. Celle-ci fut symbolisée par la référence au concept de Terre-mère, initialement porté par les organisations nord et sud-américaines puis verbalisé par le terme andin pachamama. L'instruction des situations engagées par les politiques d'assimilation, d'expropriation et d'aliénation conduisit à dénoncer le concept juridique de terra nullius (qui s'incarne en français dans l'expression "terre vacante et sans maître ») qui fonda la conquête coloniale et le concept de possession. Ce que nous dénommons le «rapport à la terre», tel qu'il est posé par le mouvement autochtone, ne s'exprime pas dans les termes contractuels de la propriété prévalant au sein du bloc occidental. Le concept de «terre» (tierra, land) est densifié par l'adjonction des notions de "territoire» (territorio, territory) et de « ressource naturelle» (recursos naturales, natural resources). Un bloc sémantique «terre, territoire et ressources» a été forgé pour permettre de penser simultanément la relation à l'environnement et la protection des personnes comme des groupes humains qui tirent leurs moyens d'existence de pratiques culturales (et culturelles) distinctes. En mobilisant le concept de Terre-mère, y compris dans les régions du monde qui ne l'énoncent pas ainsi dans leur langue, les représentants autochtones personnifient une entité objectivée dans la pensée occidentale. Ils disent «la Terre-mère souffre... », pour renvoyer à des développements sur la désertification, les désastres écologiques ou le changement climatique, et à leur devoir de la protéger. Cela nourrit un dispositif visant à établir le rôle unique des cultures, savoirs, savoir-faire, connaissances traditionnelles et spiritualités autochtones, dans la protection de l'humanité tout entière. 
Notre Terre-mère souffre et nous en sommes tous responsables. Mais comment aider la Terre lorsque chaque acteur joue un rôle mais ne s'y tient pas? Comment faire, lorsque chacun de nous doit agir mais ne le fait pas? Il est temps d'agir. ${ }^{10}$

Nous n'évoquons ces éléments, sous une forme trop synthétique et ne rendant pas justice à la nature des débats engagés sur ces questions, que pour signaler l'existence d'une articulation efficace entre la scène onusienne dédiée à l'examen et à la protection des droits des peuples autochtones et d'autres scènes globales dédiées à l'examen et à la protection de la diversité biologique ou de la propriété intellectuelle. Car le dispositif propice à la reconnaissance des peuples autochtones a des effets dans d'autres dispositifs dans lesquels les délégués autochtones sont amenés à jouer un rôle.

C'est à ce titre que la Déclaration des Nations unies sur les droits des peuples autochtones devient un symbole puissant. Dans l'imaginaire collectif des autochtones, elle fonctionne comme l'équivalent de la Charte des Nations unies et l'équivalence prit la forme d'une expression « Nous, peuples autochtones... », verbalisée dans les déclarations de caucus et postée sur différents sites web.

\section{L'institutionnalisation des questions autochtones et le partage du monde}

La montée en visibilité des problématiques autochtones s'est appuyée sur une dynamique de participation et d'alliance avec la société civile d'une part, les secteurs onusiens d'autre part, donnant lieu à un processus d'institutionnalisation unique. Celui-ci s'est caractérisé par la création du premier Groupe de travail sur les populations autochtones (1982-2006) qui servit de tribune aux autochtones du monde (Bellier, 2006b) où, en exprimant des doléances relatives aux violations des droits de l'homme, ils expérimentèrent les formes du dialogue onusien et contribuèrent à l'élaboration du projet de Déclaration.

Ce lieu, quasiment matriciel, fut doublé à partir de 1995 par un autre Groupe de travail, destiné à négocier le langage de la Déclaration, qui positionna l'ensemble autochtone par rapport aux États. Ceux-ci étaient, entre autres, organisés en cinq blocs régionaux, hérités de processus antérieurs et externes aux problématiques autochtones : Afrique, Amérique latine et Caraïbes, Asie, Europe occidentale et reste du monde, Europe orientale et ancienne Union Soviétique. Les organisations autochtones formèrent leurs propres caucus régionaux (au nombre de sept) pour passer des alliances et entamer le lobbying des États. Elles organisèrent leur travail sur ces deux plans, pour engager dans la négociation un bloc d'organisations régionales - lesquelles se structu-

10. Intervention de Candido Mezua Salazar, représentant autochtone de Panamá au Sommet sur les paysages du monde, 2014, https://ciseiweb.wordpress.com/2014, consulté le 23 mars 2015. 
rèrent à cette époque - qui se rejoignent dans une structure dénommée global indigenous caucus. Ces blocs déterminent le choix de porte-parole capables de porter la voix de l'ensemble des peuples autochtones dans l'enceinte de l’Assemblée générale.

Dans le courant des années quatre-vingt-dix, les Nations unies adoptèrent la Journée internationale (9 août) puis l’Année internationale des populations autochtones (1993), une première Décennie internationale sur le thème du «Partenariat dans l'action» (1995-2004) et une seconde Décennie sur le thème du «Partenariat pour l'action et la dignité » (2005-2014). Trois institutions prirent corps au début des années deux mille : l'Instance permanente sur les questions autochtones et le rapporteur spécial sur les droits et libertés fondamentales des peuples autochtones (en 2001) ; le Mécanisme d'experts sur les droits des peuples autochtones viendra, à partir de 2008, positionner l'interaction des autochtones avec le Conseil des droits de l'homme et le dispositif de l'Examen périodique universel.

Les premières décisions marquent l'adoption des peuples autochtones par la communauté internationale. Les institutions furent, elles, l'objet d'une appropriation symbolique par les autochtones qui se virent non seulement élevés dans la hiérarchie onusienne, ces organes étant placés près du Conseil économique et social et près du Conseil des droits de l'homme, mais aussi reconnus dans leurs compétences. Aujourd'hui, les experts traitant des questions autochtones ne sont plus simplement des représentants des États sensibilisés aux droits de l'homme, des autochtones font désormais partie du dispositif. Le symbole est fort. Le changement s'accompagne de la mise à disposition des biographies des figures du mouvement international, dont la représentation aux Nations Unies est organisée sur la base d'une nouvelle division du monde, en sept «régions socioculturelles» (ainsi désignées par le Secrétariat de l'Instance) ${ }^{11}$ : Afrique, Amérique latine et Caraïbes, Amérique du Nord, Arctique, Asie, Océanie, Europe centrale - orientale - Transcaucasie - Fédération de Russie (Bellier, 2013a).

\section{Tensions sur l'usage des symboles : la nature et les limites de l'alliance entre la communauté internationale et les peuples autochtones}

Les symboles évoqués ont permis aux peuples autochtones de reformuler le lien qu'ils avaient avec les Nations unies - qui jusque dans les années soixante

11. Ce découpage, inspiré par les logiques organisationnelles des peuples autochtones, ne laisse pas de nous interroger sur le sens et la portée de l'attribut « socioculturel » par lequel l'Onu légitime leur représentation. 
/ soixante-dix les considéraient comme des «populations arriérées »12 - et avec le système institutionnel international. Ces peuples ont trouvé en l'Onu un allié susceptible de faire pression sur les États, de produire et de diffuser de l'information à grande échelle. La conjonction - attestée par l'institutionnalisation évoquée plus haut - a rendu possible l'intervention dans un domaine subissant un véritable rejet de la part des États membres qui en réclament le monopole : ils considèrent en général que les populations autochtones relèvent de leurs affaires intérieures. Dans les secrétariats et bureaux de l'Onu s'est engagée une nouvelle perception des problématiques à la base de ce nouveau lien, qui se diffuse dans les autres organisations internationales, qu'elles soient politiques ou financières. Les outils rhétoriques, sémantiques et visuels font partie du capital symbolique que les peuples autochtones déploient par des batailles continues, les symboles se confrontant à des questionnements provenant de plusieurs directions.

D'un côté, la production symbolique a connu certains succès aux plans politiques et institutionnels. En Amérique latine, en Afrique, en Asie, au Nord de l'Europe, des constitutions et des lois visent à reconnaître et à protéger les droits de ces populations. On vérifie ainsi que les pratiques symboliques peuvent «générer, reproduire et perpétuer, et corroborer ou étendre les sens sociaux et les objectifs dans la sphère internationale» (Krotz, 2002, p. 4). De l'autre, ces symboles, tout comme les normes qu'ils cherchent à promouvoir, trouvent des obstacles à leur diffusion. Le fait que les changements se produisent dans un contexte global (mais néanmoins situé) rend difficile la reproduction aux niveaux organisationnels et locaux de l'effet qu'ils ont dans les espaces internationaux: celui de permettre aux représentants autochtones de compter sur un droit acquis de participation. Par exemple, contrairement à la Banque mondiale et aux agences spécialisées qui font partie du système des Nations unies, l'Organisation mondiale du commerce qui focalise nombre de critiques pour les politiques de libéralisation des marchés contestées par les organisations autochtones n'est pas prête à ouvrir en son sein un espace de participation pour celles-ci. C'est l'un des principaux enjeux, alors que la dissémination des normes éthiques et des indicateurs de performance par le biais des agences internationales de développement actives sur le terrain montre l'émergence d'une forme transnationale de gouvernementalité.

12. La Convention 107 de l'OIT relative aux populations aborigènes et tribales (1957) décrit les «populations tribales ou semi-tribales dans les pays indépendants» comme vivant dans des conditions sociales et économiques «correspondant à un stade moins avancé que le stade atteint par les autres secteurs de la communauté nationale». L'Inde dispose toujours d'une catégorie dite des « castes arriérées » (backward casts). 


\section{La nature intergouvernementale de l'Onu}

Les premiers représentants autochtones qui ont visité Genève, en 1923 et 1925, n'ont pas pu présenter devant les États membres de la Société des Nations le droit à l'autodétermination que leurs nations réclamaient. Ils furent bloqués par le Royaume-Uni. Une autre dynamique a été possible dans les années soixante-dix lorsque les peuples autochtones se sont présentés comme des groupes discriminés et avec le soutien d'organisations de la société civile. Le système onusien a amendé ses règles d'accréditation des organisations pour autoriser la plus large participation des autochtones. Mais la nature intergouvernementale de l'Onu se heurte toujours à l'affirmation des peuples autochtones comme sujets de droit international, et ne permet pas leur représentation autrement que par le moyen des ONG. C'est ainsi que plusieurs chefs amérindiens demandent de voir accréditées les autorités représentatives de leurs nations et non les ONG qu'elles ont mises en place pour accéder à l'Onu.

Sur la scène des Nations unies, la distinction entre les États et les organisations représentatives des peuples autochtones est visible, comme est perceptible le différentiel de pouvoir. On le note dans le protocole qui régit l'organisation des séances de discussion. Les États ont la priorité pour prendre la parole et le contenu des interventions autochtones est sous contrôle, au risque, pour les délégués qui passeraient les limites du «discours acceptable », d'être exclus de la salle. L'acceptabilité passe par la maîtrise des codes du discours international, notamment dans les critiques adressées à l'État, celui-ci disposant d'un droit automatique de réponse et de moyens pour désigner les représentants momentanément «indésirables». Les peuples autochtones partagent avec l'Onu - par l'entremise des secrétariats et des experts - le pouvoir d'élaborer des normes, mais ils ne participent pas à la prise de décision qui appartient aux États membres, sur le principe «un pays, une voix». Leurs avancées dépendent des alliances passées avec des États qui répercutent leurs positions, ainsi par exemple des États scandinaves ou latino-américains, dits «amis».

La normalisation que stimule l'inscription onusienne des peuples autochtones se retrouve dans le cadre du «partenariat» établi par les programmes et les commémorations internationales qui leur sont dédiées. Cela a certaines conséquences quant à l'autonomie et à la radicalité des propositions autochtones. Après une période d'affirmation dans les années quatre-vingt-dix et 2000, différents moyens visent à réduire le poids des peuples autochtones, mis sur le même plan que d'autres acteurs. En 1992, lors du Sommet de la terre à Rio de Janeiro, les autochtones acquirent la possibilité d'être considérés comme l'un des neuf Groupes majeurs de la société civile (avec les femmes, les enfants et la jeunesse, les syndicats, les autorités locales, les paysans, les entreprises et l'industrie, les sciences et technologies et les ONG). Ce dispositif, 
qui autorise certaines modalités de dialogue avec les chefs d'État et d'articulation avec la communauté internationale, est contesté pour plusieurs motifs que nous n'examinerons pas ici, y compris par les acteurs autochtones pour ses effets tendant à diluer les problématiques qu'ils portent.

Le symbolisme de l'égalité, qui succède à la période d'expression d'une spécificité des peuples autochtones, permet en effet d'évacuer les problématiques économiques et politiques dures qui se trouvent en jeu dans le rapport entre les peuples autochtones et les États qui les englobent. C'est ainsi, par exemple, que dans le dispositif du développement durable, les leaders autochtones refusent de voir leurs problématiques diluées dans l'usage de l'expression «communautés locales et traditionnelles»; ces entités ne jouissent en effet d'aucun droit reconnu en droit international et ne peuvent pas, en particulier, faire jouer le droit à la consultation prévu pour les peuples autochtones par la Convention 169 de l'OIT13, ni le droit au consentement prévu par la Déclaration.

Si l'on note plusieurs évolutions des fonctions de l'État par rapport aux «questions autochtones », l'État garde le monopole sur la propriété des sols et du sous-sol ainsi que la liberté de concéder l'usage des ressources naturelles et d'autres domaines, ce qui n'est pas sans effet sur la garantie des droits des peuples autochtones. Certes, ce monopole est entamé par les moyens dont disposent les entreprises multinationales pour obtenir des concessions d'exploitation et par le déploiement des cadres juridiques contraignants que sont les Conventions internationales. Mais les questions territoriales sont devenues une source majeure de contentieux porté devant les tribunaux nationaux, puis devant les Cours régionales des droits de l'homme (voir, dernièrement, le cas Saramaka vs Suriname traité par la Cour interaméricaine des droits de l'homme, et le cas Endorois vs Kenya traité par la Commission africaine sur les droits de l'homme et des peuples). Et on constate que si l'Onu a gagné un rôle de gouvernance des "questions autochtones » sur lequel s'appuient les autochtones en ayant recours aux organes de traités, les États ne sont pas tous prêts à reconnaître que les droits des peuples autochtones relèvent des juridictions du droit international, ni à répondre de bonne foi aux enquêtes des Cours régionales de droits de l'homme.

\section{Les tensions institutionnelles}

La dynamique du partenariat inclut un nombre toujours plus grand de personnes autochtones dans les consultations politiques, mais plusieurs tensions témoignent des limites posées par la fragmentation de l'Onu en une multiplicité d'organes et par la non-extension du dispositif onusien aux autres acteurs

13. La Convention 169 de l'OIT relative aux droits des peuples autochtones et tribaux reconnaît des droits collectifs aux terres, à la participation et à la consultation, ainsi que des obligations pour l'État d'adopter des mesures spéciales lorsque ses politiques visent ces populations. Elle concerne les programmes des agences spécialisées de l’Onu sur le terrain. 
internationaux. Il en résulte une discontinuité du flux des symboles dans le scénario international.

Le mouvement autochtone a visé l'instauration dans l'Onu d'espaces dédiés au traitement de leurs demandes. En parallèle, la politique de mainstreaming de cette organisation (Bellier, 2013b) s'efforce d'intégrer les «questions autochtones » dans tous les politiques et programmes d'action. Des mécanismes de coordination ont été mis en place avec l'établissement de "points focaux» dans les agences spécialisées, la création d'un Groupe de soutien inter-agences, la mise en œuvre d'un programme d'action dénommé «Partenariat des Nations unies et des peuples autochtones », doté par les contributions volontaires des États. Cette politique produit une autre série d'espaces cloisonnés qui, certes, se dédient aux questions autochtones mais ne concernent qu'une partie du programme d'action des différentes organisations.

Le rapport de force entre les peuples autochtones et les institutions internationales ne permet pas aux organisations autochtones de faire une contribution significative (ou systématique) à la compréhension des différents champs de compétence des agences spécialisées. Ce que les délégués autochtones entendent par «éducation », «alimentation » ou « santé » peut se révéler très différent de ce que l'Unesco, la FAO ou l'OMS conceptualisent - au contraire de ce qui s'est passé dans les secteurs des droits de l'homme, où un véritable débat sur les fondements du droit international eut lieu au moment de rédiger la Déclaration. Pour certains militants autochtones, la Déclaration doit maintenant permettre d'interpréter ou de faire évoluer les mandats des agences spécialisées. Pour éclairer cet aspect, signalons qu'en 1999, l'Unesco organisa une réunion sur les enjeux culturels de la Décennie internationale des populations autochtones du monde ${ }^{14}$. Pour les militants présents à cette réunion, la protection de la diversité qu'incarnent les peuples autochtones devait s'appuyer sur une compréhension du trinôme langue-culture-terre, afin de respecter le droit à l'autodétermination. Cela remettait en question l'approche sectorielle, basée sur l'individu, que défendait l'Unesco ; l'organisation évolua pour ouvrir de nouveaux projets sur les «savoirs autochtones», ou sur la «cartographie culturelle».

Face à la fragmentation institutionnelle qui a pour résultat des contradictions entre des politiques sectorielles, les organisations autochtones revendiquent une approche holistique, intégrale, coordonnée. Elles font du socle des droits fondamentaux le pilier de leur activisme. Ce faisant, elles doivent composer avec les résistances héritées des manières nationales de considérer la question autochtone. On a pu observer ces tensions dans les discussions relatives à l'agenda post-2015 qui prend la suite des Objectifs du Millénaire pour le développement, et lors de la Conférence mondiale des peuples autochtones, placée sous l'égide de l'Assemblée générale (septembre 2014). 
Des limitations ont été posées aux conditions de participation, obligeant chaque région du monde à sélectionner, en quelques jours, une trentaine de personnes autochtones représentatives : un défi très difficile à relever. Les dispositifs très ouverts, pratiqués au Groupe de travail sur les populations autochtones, à la Convention sur la diversité biologique ou à la Commission du développement durable, ne sont pas admis partout. Cela a pour premier effet de conforter la professionnalisation de quelques acteurs qui s'appuient sur leurs liens privilégiés avec l'ONU pour disposer de l'information et bénéficier de soutiens, notamment financiers, et pour effet secondaire de diviser le mouvement international qui, ne bénéficiant pas des moyens dont disposent les appareils d’État pour assurer leur représentation, fait face à des compétitions internes délicates à gérer.

Par ailleurs, les banques de développement et les compagnies extractives qui sont l'objet de critiques récurrentes ont élaboré leurs propres références pour ne pas adopter les références onusiennes plus ambitieuses. La Banque mondiale, responsable de projets de développement dénoncés pour ne pas respecter les droits des communautés locales, offre ainsi une définition de travail à travers laquelle elle cherche, par l'usage de l'expression «populations autochtones », à atténuer la reconnaissance des droits des peuples autochtones (et ses effets juridiques) dans la communauté internationale. Elle a subtilement opposé au concept de « consentement libre, préalable et éclairé/ informé », revendiqué par les autochtones et acté dans la Déclaration, le principe de «consultation préalable, libre, et fondée sur la communication des informations nécessaires »15. Or le lien entre "consultation » et «consentement » est posé par la Convention $169^{16}$. La nuance introduite et défendue par la Banque mondiale n'échappera pas aux juristes.

\section{Ce que les symboles cachent}

La mobilisation des outils rhétoriques et sémantiques - qui vont au-delà du symbole qu'ils semblent être - constitue la base des négociations que les délégués autochtones entreprennent pour établir de nouvelles relations au niveau international, national et local. En se servant de ces symboles dans leurs différentes négociations, le mouvement autochtone diffuse plus que ses revendica-

15. Manuel opérationnel de la Banque mondiale. Politiques opérationnelles 4.10 sur les populations autochtones, 2005. Voir p. 1, note 4 : «Ce processus ne confère pas de droit de veto individuel ou collectif. » La banque conditionne le résultat de la consultation et l'obtention du consentement à un vote positif. Or les autochtones réclament le droit de pouvoir dire oui ou non. Voir http://siteresources.worldbank.org/OPSMANUAL/Resources/OP4.10.July1.2005.French.pdf, consulté le 2 février 2015.

16. L'article 6, alinéa 2, de la Convention 169 stipule : "Les consultations effectuées en application de la présente convention doivent être menées de bonne foi et sous une forme appropriée aux circonstances, en vue de parvenir à un accord ou d'obtenir un consentement au sujet des mesures envisagées. » (Souligné par les auteurs) 
tions. Mais il ne peut éviter de satisfaire aux critères politiques, économiques et légaux de l'Onu. C'est ainsi que les transactions au nom des droits autochtones occultent des valeurs précises, en provenance de la culture occidentale, qui s'installent dans les communautés autochtones du monde.

Cet aspect représente le coût (que l'on mesurera dans plusieurs années) de l'alliance internationale pour les communautés autochtones. Parmi les éléments de coût, nous identifions : les changements qui se produisent dans les structures de pouvoir, entraînés par la création d'ONG - pour capter des financements ou sceller un partenariat avec une agence locale de développement et par la formation de personnes qui ne correspondent pas aux critères de gouvernement propre pour la chefferie ou le leadership; les nouveaux principes de distribution des terres et d'enregistrement des droits; l'émergence d'un ordre symbolique fondé sur la rhétorique des droits de l'homme et de nouveaux modèles de relations au sein des communautés (de genre, et transgénérationnels) ainsi que sur la référence au marché.

Pour le mouvement autochtone international, l'impact que les organisations internationales ont dans les communautés plus «sensibles culturellement» ou plus menacées par les nouvelles formes de la globalisation, devient un front de lutte. Les «autochtones onusiens», reconnus experts, semblent ainsi obéir à un double objectif, à savoir de conserver la place acquise au niveau international sans sacrifier les «cultures ». Cette position illustrée par les collaborations récentes avec l'Unesco ou la FAO vise à améliorer les indicateurs (de la diversité, des systèmes agroalimentaires ou de la gouvernance).

Mais la fonction de protection des symboles qui donnent du pouvoir (empowerment - qui s'incarne dans l'expression récente de «Rien sur nous sans nous ") ${ }^{17}$ se heurte au fait que, en matière autochtone comme ailleurs, la gouvernance s'appuie sur une technicisation qui laisse de côté la plupart des aspects politiques. Les peuples autochtones ont réussi à faire passer l'idée qu'ils subissent une discrimination, mais ils ne peuvent pas résoudre les problèmes que pose la [non-]transposition nationale des décisions prises en matière de droits au niveau international.

La coproduction symbolique que nous avons mise en avant est une particularité du système onusien qui a singularisé le domaine des droits des peuples autochtones. Elle a changé la perception des peuples autochtones dans la communauté internationale et sensiblement augmenté leur poids de négociation au niveau international et local. Les symboles mis en évidence - catégorie de nomination, outil juridique, images et formes discursives - permettent aux

17. Cette position s'est affirmée lors de la préparation de l’organisation de la Réunion plénière de haut niveau connue comme Conférence mondiale des peuples autochtones (septembre 2014) dont l’intitulé évoque la tension imposée aux procédures de l'Assemblée générale des Nations unies pour accommoder l'objectif de «participation autochtone». 
peuples autochtones de se distinguer d'autres groupes qui portent leurs intérêts devant l'ONU et d'utiliser cette distinction comme la base de ressources politiques et institutionnelles. La diffusion globale de ces symboles rencontre toutefois des réticences qu'il convient d'avoir à l'esprit quand il s'agit de faire le bilan de l'efficacité politique d'une dynamique qui visait à dépasser la logique hiérarchique des États pour doter les peuples - invisibilisés par divers processus de domination et de colonisation - de nouvelles formes de pouvoir (empowerment).

Le manque de transparence des aspects politiques qui entrent en jeu dans la circulation des symboles mentionnés demeure une source de contentieux, que l'on observe plus au niveau local des communautés qu'au niveau international des dialogues diplomatiques. Au-delà du symbole, la question fondamentale reste de garantir les droits de ces populations dans un ensemble de dispositifs d'où elles sont encore exclues (non-accès à la citoyenneté, exclusion sociale, marginalisation économique). Cela engage les organisations internationales qui les reconnaissent, mais aussi l'État qui reste un moyen clé pour l'organisation et l'opérativité des accords internationaux. C'est ainsi que même à partir d'une enceinte internationale comme celle de l'ONU, aussi ouverte et bien disposée soit-elle à l'égard des peuples autochtones, on ne peut éviter de tenir compte des jeux politiques nationaux et locaux dont dépend de facto le devenir des peuples autochtones. Il faut donc mieux comprendre la nature de la relation tripartite qui s'est établie pour envisager l'achèvement des compromis exprimés dans les symboles convenus entre les peuples autochtones et l'ONU.

\section{Références}

Allen Stephen, XANTHAKI Alexandra éd., 2011, Reflections on the UN Declaration on the Rights of Indigenous Peoples, Oxford, Hart Publications.

ANDERSON Benedict, 1991, Imagined Communities. Reflections on the Origin and Spread of Nationalism, revised edition, New York, Verso.

Baldwin Clive, Morel Cynthia, 2011, "Using the United Nations Declaration on the Rights of Indigenous Peoples in litigation », Reflections on the UN Declaration on the Rights of Indigenous Peoples, S. Allen, A. Xanthaki éd., Oxford, Hart Publications, p.121-146.

BELLIER Irène, 2005, "The Declaration of the Rights of Indigenous Peoples and the World Indigenous Movement », Griffith Law Review, nº 14 (2), p. 227-246.

- 2006a, "Le projet de déclaration des droits des Peuples autochtones et les États américains. Avancées et clivages", Être indien dans les Amériques, C. Gros, M.-C. Strigler éd., Paris, Institut des Amériques, p. $27-42$.

— 2006b, "Les Peuples autochtones à l'Onu. Genèse d'une identité globale, avatars régionaux et logiques représentatives ", L'identité en miettes. Limites et beaux 
risques politiques aux multiculturalismes extrêmes, P. Boudreault éd., Paris, L'Harmattan, 2006, p. 71-88.

- 2007, «Partenariat et participation des Peuples autochtones aux Nations unies. Intérêt et limites d'une présence institutionnelle», C. Neveu éd., Démocratie participative, cultures et pratiques, Paris, L'Harmattan. p.175-192.

- 2012, "Les Peuples autochtones aux Nations unies. La construction d'un sujet de droits / acteur collectif et la fabrique de normes internationales», Critique internationale, $\mathrm{n}^{\circ} 54$, p. 61-80.

- 2013a, "La reconnaissance internationale des Peuples autochtones», Peuples autochtones dans le monde. Les enjeux de la reconnaissance, I. Bellier éd., Paris, L'Harmattan, p.13-37.

— 2013b, « «We, Indigenous peoples...» Global activism and the emergence of a new collective subject at the United Nations », The Gloss of Harmony. The Politics of PolicyMaking in Multilateral Organizations, B. Müller éd., Londres, Pluto Press, p. 177-201.

BRYSK Alison, 1995, «"Hearts and minds". Bringing symbolic politics back in », Polity, n०27 (4), p. 559-585.

Charters Claire, Stavenhagen Rodolfo, 2009, Making the Declaration Work. The United Nations Declaration on the Rights of Indigenous Peoples, Copenhague, IWGIA.

CRAWHALl Nigel, 2011, "Africa and the UN Declaration on the Rights of Indigenous Peoples », The International Journal of Human Rights, no 15 (1), p. 11-36.

DAES Erica-Irene A., 2004, Souveraineté permanente des peuples autochtones sur les ressources naturelles, Document Onu E/CN.4/Sub.2/2004/30.

- 2008, «Indigenous Peoples. Keepers of our Past-Custodians of our Future», Copenhague, IWGIA.

DAHLJens, 2009, IWGIA. A History, Copenhague, IWGIA.

FAO, 2009, Cultural Indicators of Indigenous Peoples' Food and Agro-Ecological Systems, Document Onu E/C.19/2009/CRP. 3.

HARTLEY Jackie, Joffe Paul, Preston Jennifer, 2010, Realizing the UN Declaration on the Rights of Indigenous Peoples. Triumph, Hope, and Action, Saskatoon, Purich Publishing.

KROTZ Ulrich, 2002, Social Content of the International Sphere. Symbols and Meaning in Franco-German Relations, Program for the Study of Germany and Europe, Working Paperno02.2.

MARTINEZ M. Miguel Alfonso, 1999, Étude des traités, accords et autres arrangements constructifs entre les États et les populations autochtones, Document Onu E/CN.4/ Sub.2/1999/20.

MORIN Françoise, 2006, "Les Nations unies à l'épreuve des peuples autochtones», Être indien dans les Amériques, C. Gros, M. C. Strigler éd., Paris, Institut des Amériques et Institut des Hautes études de l'Amérique latine, p. 43-54.

Muehlebach Andrea, 2001, «Making place at the United Nations. An anthropological inquiry into the United Nations Working Group on Indigenous Populations », Cultural Anthropology, no 16 (3), p. 415-435.

NIEZEN Ronald, 2003, The Origin of Indigenism. Human Rights and the Politics of Identity, Berkeley, The University of California Press. 
IRÈne BeLlier, Veronica GonzÁlez-González

PRICE Richard, 2012, Peuple Saramaka contre État du Suriname. Combat pour la forêt et les droits de l'homme, Paris, Karthala IRD.

UnesCo, 2003, Cultural Diversity and Biodiversity for Sustainable Development, Rapport d'une table ronde de haut niveau organisée par l'Unesco et l'Unep lors du Sommet sur le développement soutenable en 2002, Unesco/Unep.

Willemsen DiAz Augusto, 2013, «Comment les droits des peuples autochtones sont entrés à l'ONU », La Déclaration des droits des peuples autochtones. Genèse, enjeux et perspectives de mise en œuvre, C. Charters, R. Stavenhagen éd., Paris, L'Harmattan, p. 20-35. 